\title{
Diagnostic Challenges in Pediatric Hemophagocytic Lymphohistiocytosis
}

\author{
Stephanie J. Si ${ }^{1,2,3,4}$ - Sarah K. Tasian ${ }^{1,2} \cdot$ Hamid Bassiri $^{1,5} \cdot$ Brian T. Fisher $^{1,5} \cdot$ Jasmyn Atalla $^{6} \cdot$ Reema Patel $^{1,2}$. \\ Neil Romberg ${ }^{1,7}$ - Michele P. Lambert ${ }^{1,8}$ - Michele Paessler ${ }^{1,9} \cdot$ Edward J. Behrens ${ }^{1,7}$ - David T. Teachey ${ }^{1,2}$. \\ Kathleen E. Sullivan ${ }^{1,10}$
}

Received: 16 November 2020 / Accepted: 15 March 2021 / Published online: 24 March 2021

(C) The Author(s), under exclusive licence to Springer Science+Business Media, LLC, part of Springer Nature 2021

\begin{abstract}
Hemophagocytic lymphohistiocytosis (HLH) is a syndrome of severe immune dysregulation that encompasses a broad range of underlying genetic diseases and infectious triggers. Monogenic conditions, autoimmune diseases, and infections can all drive the phenotype of HLH and associated immune hyperactivation with hypercytokinemia. A diagnosis of HLH usually requires a combination of clinical and laboratory findings; there is no single sensitive and specific diagnostic test, which often leads to "diagnostic dilemmas" and delays in treatment initiation. Ferritin levels, one of the most commonly used screening tests, were collected across a large tertiary care pediatric hospital to identify the positive predictive value for HLH. Herein, we present several cases that illustrate the clinical challenges of confirming an HLH diagnosis. Additionally, we report on the utility of establishing a formal multi-disciplinary group to aid the prompt diagnosis and treatment of patients presenting with HLH-like pathophysiologies.
\end{abstract}

Keywords Ferritin · hemophagocytic lymphohistiocytosis (HLH) · immune dysregulation [3-6]

\section{Introduction}

HLH was first recognized as a disease of severe immune dysregulation in 1952, when the central features of fever, hemophagocytosis, hepatosplenomegaly, and progressive cytopenias were described [1]. Decades later, these sentinel features still represent cornerstone clinical indications to pursue further HLH diagnostic evaluations. Although HLH was initially characterized as a genetic disorder, we now know that

Stephanie J. Si

ssi@cc.hawaii.edu

1 Department of Pediatrics, University of Pennsylvania Perelman School of Medicine, Philadelphia, PA, USA

2 Division of Oncology and Center for Childhood Cancer Research, Children's Hospital of Philadelphia, Philadelphia, PA, USA

3 Division of Pediatric Oncology-Hematology, University of Hawai'i Cancer Center, Kapi'olani Medical Center for Women and Children, Honolulu, HI, USA

4 University of Hawai'i School of Medicine, 701 Ilalo St, Honolulu, HI 96813, USA this disease can also occur sporadically, often in association with infections, malignancies, or rheumatologic and autoinflammatory disorders. The etiologies of HLH differ between adults and children, but both populations display a number of conditions that could result in HLH [2].

Familial HLH is defined as immune dysregulation that is driven by mutations in one of four genes (PRF1, UNC13D, $S T X 11$, and $S T X B P 2$ ) that encode proteins involved in vesicle formation and trafficking [3]. These four monogenic conditions

5 Division of Infectious Diseases, Children's Hospital of Philadelphia, Philadelphia, PA, USA

6 Department of Internal Medicine and Department of Pediatrics, Vidant Medical Center, East Carolina University, Greenville, NC, USA

7 Division of Rheumatology, Children's Hospital of Philadelphia, Philadelphia, PA, USA

8 Division of Hematology, Children's Hospital of Philadelphia, Philadelphia, PA, USA

9 Department of Pathology, Children's Hospital of Philadelphia, Philadelphia, PA, USA

10 Division of Allergy and Immunology, Children's Hospital of Philadelphia, Philadelphia, PA, USA 
have a high penetrance, and patients are often prone to disease recurrence ("flares") until they undergo immune system replacement with allogeneic hematopoietic stem cell transplant (HSCT). Several other primary immunodeficiencies also have a high rate of HLH, including Chediak-Higashi syndrome due to mutations in LYST, Griscelli syndrome due to mutations in $R A B 27 A$, and Hermansky-Pudlak syndrome type 2 due to mutations in $A P 3 B 1$, which collectively comprise the pigmentary dilution conditions associated with HLH. Other primary immunodeficiencies also have significant association with HLH, and treatment approaches are usually directed against the specific underlying immune defect [4].

In contrast, in the absence of an identified genetic cause, secondary or acquired HLH can be triggered by severe infections, autoimmunity, or malignancy [5]. Such patients often represent a diagnostic conundrum, as infectious etiologies can also trigger the first manifestations of primary or familial HLH. Moreover, HLH occurring in the setting of nonmonogenic rheumatologic or autoinflammatory disease is often termed macrophage activation syndrome (MAS) [6]. HLH-like processes can also occur in the setting of metabolic conditions such as lysinuric protein intolerance, Wolman syndrome, and Gaucher's disease; treatment in these cases relies upon correction of underlying metabolic imbalances. Finally, certain immunotherapies used for cancer treatment, including chimeric antigen receptor (CAR) T cells, can lead to cytokine release syndrome (CRS), which clinically and biologically is diagnosed as HLH when severe. As such, classification of primary versus secondary HLH can be complicated given the myriad of potential triggering stimuli, heterogeneity of underlying genetic causes, and the clinical overlap in the pathophysiologic presentations of these diseases.

While no single diagnostic test exists for HLH, a set of criteria was recommended by the Histiocyte Society for use in the HLH-2004 research protocol and was revised in 2007. These criteria include identification of an HLH-associated gene defect and/or the presence of at least five of the following eight criteria: (1) fever, (2) low or absent natural killer (NK) cell function, (3) cytopenias, (4) splenomegaly, (5) increased triglycerides or low fibrinogen, (6) high ferritin, (7) hemophagocytosis, and (8) elevated soluble CD25 (interleukin 2 receptor alpha (IL2R $\alpha)$ ). Among patients with validated $\mathrm{HLH}$, each criterion has a frequency of 71-95\% [7] but individually has a low specificity for HLH $[8,9]$. To better understand the diversity of conditions associated with increased ferritin, a key indicator of HLH, we used data from a structured database we established as part of the multi-disciplinary Immune Dysregulation program that was founded at our institution in 2018. This team includes pediatric geneticists, hematologists, immunologists, neurologists, oncologists, pathologists, rheumatologists, and infectious diseases specialists. We observed low positive predictive value for hyperferritinemia in pediatric HLH, and we present several cases in which the underlying diagnosis represented a particular diagnostic challenge in order to emphasize the diversity of conditions with clinical overlap with HLH.

\section{Methods}

This report is a single-institution retrospective chart review of patients with markedly elevated ferritin levels admitted to the Children's Hospital of Philadelphia (CHOP) between January 1, 2019, and January 1, 2020. This study was determined to be exempt from human subjects research approval by the CHOP Institutional Review Board. This project began as a quality improvement initiative to prospectively and systematically screen all children and young adults that were seen during an inpatient or outpatient visit at CHOP who had a single or multiple elevated ferritin value. A daily EPIC query was used to generate a $\log$ of all patients with a ferritin of $>500 \mu \mathrm{g} / \mathrm{L}$. This $\log$ was reviewed daily by members of the Immune Dysregulation Team to identify patients in whom multidisciplinary consultation could have led to more timely diagnoses of HLH or other disorders of immune regulation. Data were maintained in a secure encrypted REDCap $®$ database. To study this population comprehensively and to ascertain the potential specificity of hyperferritinemia, patients were further analyzed and classified according to age, sex, ethnicity, and primary diagnosis or underlying trigger and were grouped into the following categories: HLH/MAS, allogeneic or autologous stem cell transplant, malignancy, toxicities related to immunotherapy (e.g., CRS from chimeric antigen receptor T cell therapy/bi-specific $\mathrm{T}$ cell engager antibody immunotherapy), iron overload from chronic transfusions, infection, liver failure, and other (including metabolic disorders such as methylmalonic acidemia, MDA5+ associated interstitial lung disease, and necrotizing enterocolitis).

Statistical analysis was performed using Prism 8 (Version 8.2.0, Graphpad Software, La Jolla, CA) using analysis of variance (ANOVA) testing for group comparisons. Significant results are denoted by $p$ value $<0.05$.

\section{Results}

We analyzed a total of 163 patients with ferritin $>500 \mu \mathrm{g} / \mathrm{L}$ and patients were separated into the following categories: HLH/MAS $(n=8)$, allogeneic or autologous stem cell transplant (HSCT) $(n=4)$, malignancy $(n=16 ; n=9$ hematologic malignancy, $n=7$ solid tumor), toxicities related to immunotherapy $(n=33)$, iron overload from chronic transfusions $(n=83)$, infections $(n=$ 8), liver failure ( $n=2)$, and other $(n=9)$ (Fig. 1). HLH/MAS was diagnosed based on fulfilling the HLH criteria, use of clinical judgment, serum cytokine levels and other biomarkers, and/or identification of a commonly associated HLH predisposing gene. 
Ferritin levels ranged from a low of 2010 to a high of $148,220 \mu \mathrm{g} / \mathrm{L}$. Although only 8 of 163 patients met HLH diagnostic criteria, a total of 17 patients had ferritin levels > $10,000 \mu \mathrm{g} / \mathrm{L}$ (Table 1). This includes $3 / 8$ patients with HLH, $1 /$ 4 patients post-HSCT, $1 / 13$ patients with cancer, $6 / 33$ patients treated with chimeric antigen receptor $\mathrm{T}$ cell/bi-specific $\mathrm{T}$ cell engager therapy, $3 / 83$ patients with iron overload, $2 / 8$ patients with infections, $0 / 2$ patients with liver failure, and $1 / 9$ patients in the "other" category. Ferritin of $>10,000 \mu \mathrm{g} / \mathrm{L}$ had a positive predictive value of $18 \%$ at our institution, demonstrating that marked hyperferritinemia was not a specific biomarker for HLH in a tertiary care pediatric center. Although severe hyperferritinemia was seen among patients with the various diagnoses, those with HLH/MAS had statistically significantly higher mean ferritin levels ( $p=0.005$ by one-way analysis of variance [ANOVA] ) when compared to other categories (Fig. 2).

Table 1 Maximum, average, and median ferritin levels observed across disease categories

\begin{tabular}{ll}
\hline & $\mu \mathrm{g} / \mathrm{L}$ (range) \\
\hline HLH/MAS $(n=8)$ \\
Max & $195,590(2588-195,590)$ \\
Average & 33,276 \\
Median & 6598 \\
Stem cell transplant $(n=4)$ \\
Max & $23,883(2113-23,883)$ \\
Average & 7614 \\
Median & 2230 \\
Malignancy $(n=16)$ \\
Max & $74,660(2397-74,660)$ \\
Average & 8300 \\
Median & 3020 \\
Immunotherapy & toxicities $(n=33)$ \\
Max & $64,231(2030-64,231)$ \\
Average & 7964 \\
Median & 3703 \\
Iron overload $(n=83)$ \\
Max & $19,203(2130-19,203)$ \\
Average & 3536 \\
Median & 2627 \\
Infections $(n=8)$ \\
Max & $148,220(2215-148,220)$ \\
Average & 22,152 \\
Median & 2631 \\
Liver failure $(n=2)$ \\
Max & $2944(2042-2944)$ \\
Average & 2493 \\
Median & 2493 \\
Other $(n=9)$ & $41,620(2101-41,620)$ \\
Max & 7107 \\
Average & 2525 \\
Median & \\
\hline &
\end{tabular}

\section{Illustrative Cases}

Diagnosis of HLH requires identification of five of eight clinical criteria when a causal gene mutation is not identified. As these criteria may evolve sequentially or be incomplete, there is a true art to the early diagnosis of HLH. These cases herein represent significant diagnostic challenges and are described to provide context for ongoing evolution of the diagnosis and management of HLH beyond typical known causes. Table 2 presents the diagnostic characteristics of 17 patients without familial HLH who were evaluated by our multi-disciplinary Immune Dysregulation Team when HLH became a diagnostic consideration. Not all patients fulfilled the complete classical diagnostic criteria for HLH, and only a few children received standard HLH therapy. We classify patients by their ultimate diagnoses into four categories: (1) metabolic diseases, (2) rheumatologic diseases, (3) primary immunodeficiencies, and (4) infectious etiologies where the pathogen was considered to be the driving force leading to hyperinflammation. Referrals to our Immune Dysregulation Team were made based on clinical features suggestive of HLH, while in others, laboratory features suggested the diagnosis. The ultimate identification of an etiology occurred after multi-disciplinary dialog, targeted investigations, and revisiting the evolution of patients' clinical course over time.

\section{Discussion}

The Histiocyte Society established the first set of diagnostic guidelines for HLH in 1991 [10] and the first prospective international treatment protocol (HLH-94) in 1994 [11]. Revisions to both diagnostic criteria and treatment strategies were subsequently made with inclusion of three additional diagnostic criteria in the HLH-2004 protocol, including low or absent NK cell activity, hyperferritinemia, and high levels of soluble CD25 [12]. Given the relative non-specificity of the individual criteria, a diagnosis of HLH requires that at least five of the eight criteria be fulfilled unless a molecular mutation/diagnosis consistent with HLH has been identified. To highlight the complexity of HLH diagnosis in children, we analyzed a dataset from our Immune Dysregulation study.

Hyperferritinemia can occur in various disease processes including sepsis, infection, liver failure, malignancy, and HLH [13]. Previous studies have shown that a ferritin level of $>500 \mu \mathrm{g} / \mathrm{L}$ is $>90 \%$ sensitive for HLH, but specificity is only robust at levels of $>2000$ to $10,000 \mu \mathrm{g} / \mathrm{L}$ [14]. Results from our institution confirmed this conceptual conclusion and supports the non-specificity of hyperferritinemia as a single biomarker in diagnosing HLH. As a ubiquitous iron-binding protein that regulates iron storage and homeostasis, the ferritin heavy chain gene is regulated by oxidative stress as well as pro-inflammatory signaling through the NF-kB pathway $[13$, 


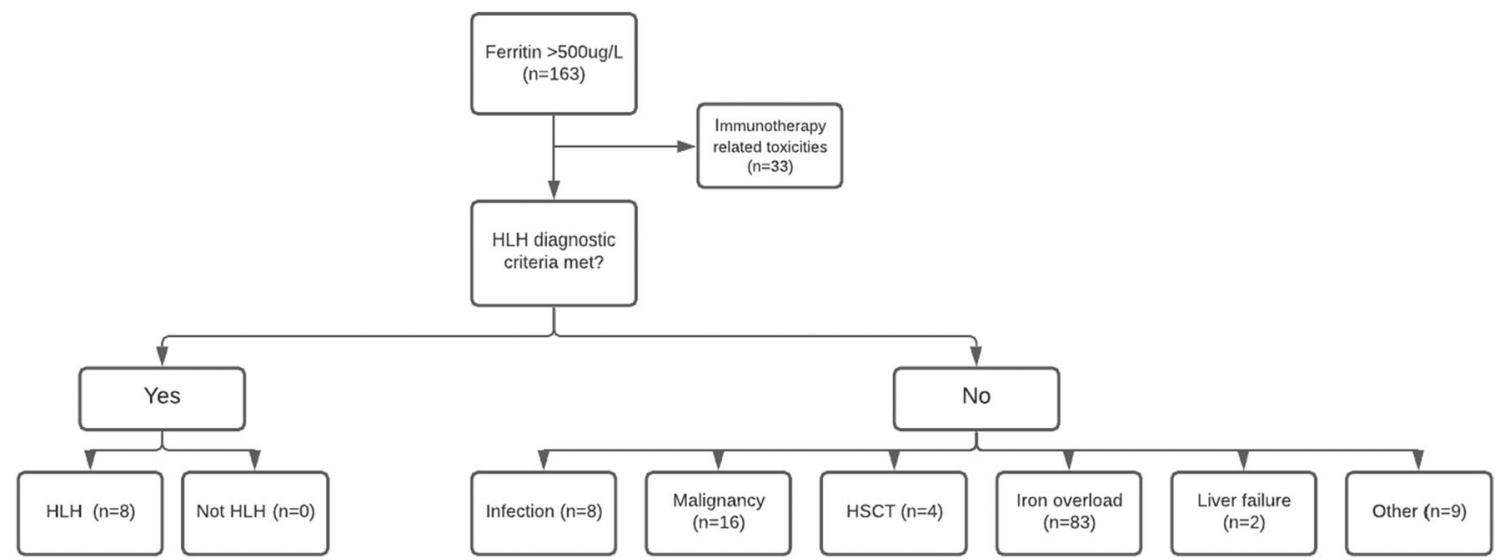

Fig. 1 Flow chart representation of various diagnoses associated with hyperferritinemia, highlighting its non-specificity as a single biomarker in diagnosing HLH

15]. Our data emphasize the range of conditions in which hyperferritinemia occurs and tabulate the sensitivity and specificity of ferritin $>10,000 \mu \mathrm{g} / \mathrm{L}$, further highlighting the many opportunities for diagnostic confusion. Our data set predates the COVID-19 pandemic, although current utilization of the hyperinflammation index to identify patients at high risk of severe diseases also relies on some of the same criteria used for diagnosing HLH $[16,17]$. While it is widely recognized that individual criteria are insensitive and non-specific for $\mathrm{HLH}$, the actual application of these composite criteria in the

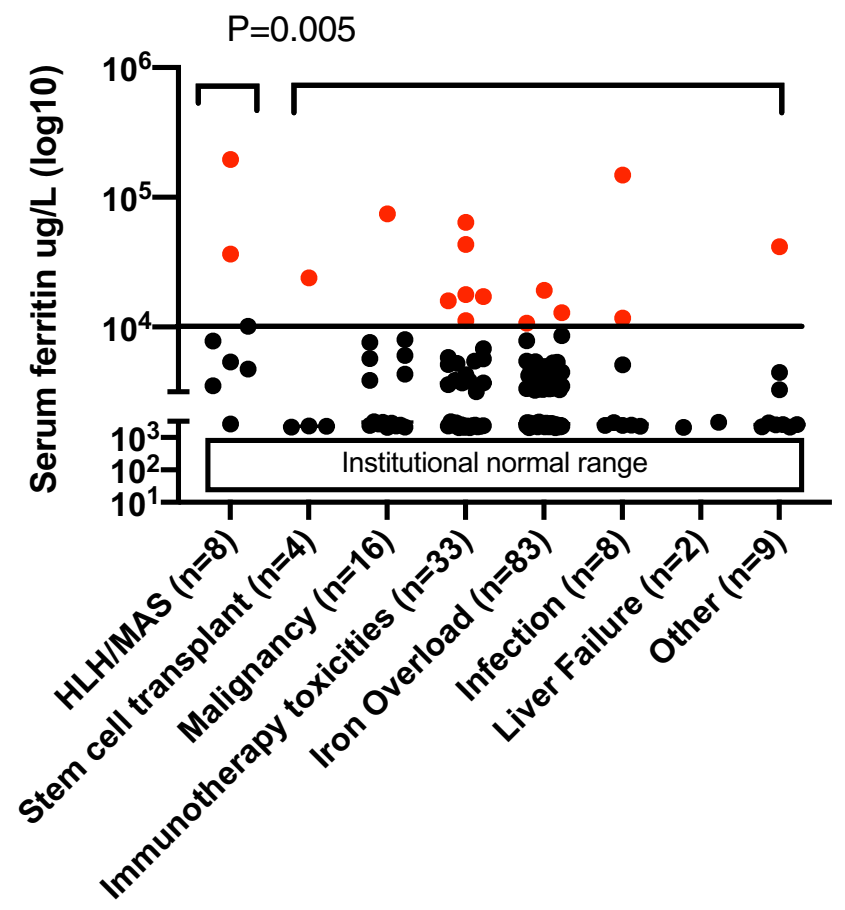

Fig. 2 Peak ferritin concentrations across disease categories. Severe hyperferritinemia were seen in various diagnoses, but patients with HLH/MAS had statistically significantly higher mean ferritin levels when compared to other categories setting of an acutely ill child where HLH is on the differential diagnosis can be quite complex. We describe illustrative patient cases with non-straightforward diagnoses to highlight the diagnostic challenge of simultaneously entertaining multiple etiologic possibilities of disease.

Secondary HLH in setting of infections, malignancies, and underlying immunodeficiency without a known monogenic cause of HLH are a particular concern, as the required treatments can be quite different. Interactions of pathogens with undefined aspects of host responses are thought to underlie HLH in these settings. Advances in our understanding immune function, along with increasingly-available scientific tools such as WES and WGS, will surely continue to expand our knowledge of HLH beyond the common gene mutations classically associated with primary HLH $[2,18]$ and identification of polymorphisms in known genes [19]. Our patient cases demonstrate the varied underlying conditions where HLH-like features can be seen and in which underlying immune compromise and/or specific infectious pathogens contributed to immune dysregulation. While allogeneic HSCT was performed for several patients to correct their underlying immune dysfunction, other children who did not undergo HSCT were treated with approaches intended to quell inflammation and allow reestablishment of immune homeostasis. In settings of acute infection, it can be challenging to distinguish whether the key driver of inflammation is an infection or whether host dysfunction is the central underlying pathologic process. In these challenging cases, our Immune Dysregulation Team, comprised of the specialties described above, initially is contacted through a rapid-response list-serve. The on-call member, a dedicated nurse practitioner, and the responding specialists will provide a response within $24 \mathrm{~h}$ for these critically ill children. The entire team reviews patients' charts in detail during a weekly multidisciplinary conference and weigh the benefits and limitations of different therapeutic approaches in these complex patients. As the patient's status evolves, the discussions continue. If 
Table 2 HLH as a manifestation

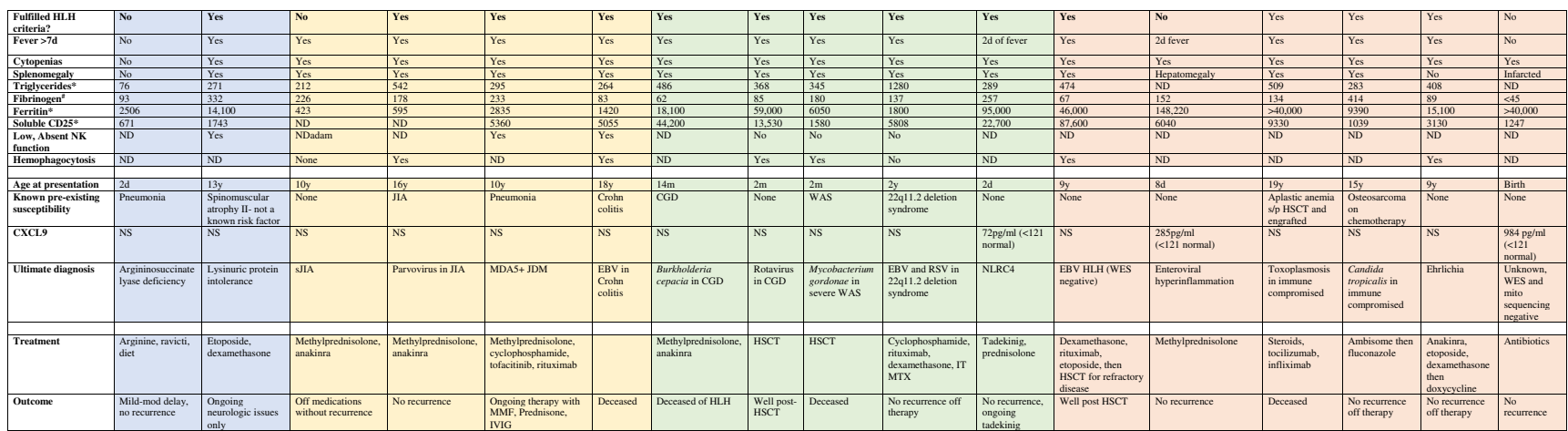

*Peak during illness

${ }^{*}$ Nadir during illness

$N D$ not done

needed, outpatient follow-up is available through our Immune Dysregulation clinic.

Treatment of patients with HLH continues to be challenging, as refinements of protocols since HLH-1994 have not improved clinical outcomes [5]. Conventional therapies to date have aimed at dampening immune activation through non-specific mechanisms and comprised of chemotherapy known to increase risk of secondary malignancy (i.e., etoposide) and high-dose dexamethasone associated with increased risk of infection, osteonecrosis, and hyperglycemia [12]. In recent years, direct targeting of disease-driving pathways and avoiding myelosuppressive effects of etoposide and global immunosuppression has become possible. One such therapy is emapalumab, a fully human anti-IFN- $\gamma$ monoclonal antibody, which was approved by the US FDA in 2018 for treatment of patients with refractory HLH after a study demonstrated an overall response rate in 17 of $27(63 \%)$ patients with recurrent or refractory primary HLH (NCT01818492) [20]. Other potential precision therapies used in patients with HLH include other biologic agents that block cytokines, including anakinra (anti-IL1) and tocilizumab (anti-IL6R) [21, 22]. Optimal therapy requires clear understanding of the etiologic process(es) driving the HLH, a non-trivial endeavor that our cases highlight. As additional small molecule inhibitors (e.g., JAK inhibitors such as ruxolitinib, tofacitnib, baricitinib, and itacitinib [23-25]) become a reality, the importance of molecular diagnostics and precision therapeutics become even more important.

Although discoveries of novel molecular pathways and use of targeted immune modulating therapies have led to significant progress in the treatment of this disease, much work remains to be done. Our case series of children with unusual clinical presentations evoking HLH highlights the remarkable biologic and immunologic heterogeneity of this condition. Development of true biomarkers and sensitive and specific diagnostic tests remain the aspiration. Until such time as real time diagnostics are available, the clinical criteria for diagnosis remain in use. This study highlights the many alternative explanations for a high ferritin and the diagnostic difficulties often seen in a tertiary care pediatric center.

Author Contribution SJS and KES were involved in analyzing data and writing the manuscript. RP acquired data, and HB, BTF, NR, MPL, MP, EJB, DTT, and KES designed this research study. SJS, SKT, HB, BTF, JA, RP, NR, MPL, MP, EJB, DTT, and KES all reviewed and edited this manuscript prior to submission. All authors had substantial contributions to this manuscript.

Funding This work was supported by the Children's Hospital of Philadelphia HLH Frontiers Program, 5R01 CA193776 (DTT), U01 CA232486 (SKT), and Department of Defense Translational Team Science award CA180683P1 (SKT). EMB is the Joseph Lee Hollander Chair in Pediatric Rheumatology. KES is the Frank R Wallace Endowed Chair in Infectious Diseases.

Availability of Data and Material The datasets generated during and/or analyzed during the current study are available from the corresponding author on reasonable request.

Code Availability Not applicable.

\section{Declarations}

Ethics Approval Not applicable.

Consent to Participate Not applicable.

Consent for Publication Yes, we consent for publication.

Conflict of Interest SKT receives research funding from Incyte Corporation and Gilead Sciences for unrelated studies and serves on the scientific advisory board of Aleta Biotherapeutics. HB is an owner of CSL Behring stock and is a consultant for Kriya Therapeutics. MPL is an advisory board member for Octapharma and Shionogi, is a consultant for Amgen, Novartis, Shionogi, Dova, Principia, Argenx, Rigel, and Bayer, and has received research funding from Sysmex, Novartis, 
Rigel, and Astra Zeneca. DTT serves on advisory boards for Amgen, La Roche, Janssen, and Sobi. BTF's institution receives funding from Merck and Pfizer for research studies, and he also serves on a Data Safety Monitoring Committee for Astellas. These studies are not related to this project. The remaining authors declare no relevant conflicts of interest.

\section{References}

1. Farquhar JW, Claireaux AE. Familial haemophagocytic reticulosis. Arch Dis Child. 1952;27(136):519-25.

2. Janka GE, Lehmberg K. Hemophagocytic syndromes-an update. Blood Rev. 2014;28(4):135-42.

3. Henter JI, Elinder G. Familial hemophagocytic lymphohistiocytosis. Clinical review based on the findings in seven children. Acta Paediatr Scand. 1991;80(3):269-77.

4. Bode SF, Ammann S, Al-Herz W, et al. The syndrome of hemophagocytic lymphohistiocytosis in primary immunodeficiencies: implications for differential diagnosis and pathogenesis. Haematologica. 2015;100(7):978-88.

5. Jordan MB, Allen CE, Weitzman S, Filipovich AH, McClain KL. How I treat hemophagocytic lymphohistiocytosis. Blood. 2011;118(15):4041-52.

6. Grom AA. Macrophage activation syndrome and reactive hemophagocytic lymphohistiocytosis: the same entities? Curr Opin Rheumatol. 2003;15(5):587-90.

7. Bergsten E, Horne A, Arico M, et al. Confirmed efficacy of etoposide and dexamethasone in HLH treatment: long-term results of the cooperative HLH-2004 study. Blood. 2017;130(25):272838.

8. Ho C, Yao X, Tian L, Li FY, Podoltsev N, Xu ML. Marrow assessment for hemophagocytic lymphohistiocytosis demonstrates poor correlation with disease probability. Am J Clin Pathol. 2014;141(1):62-71.

9. Goel S, Polski JM, Imran H. Sensitivity and specificity of bone marrow hemophagocytosis in hemophagocytic lymphohistiocytosis. Ann Clin Lab Sci. 2012;42(1):21-5.

10. Henter JI, Elinder G, Ost A. Diagnostic guidelines for hemophagocytic lymphohistiocytosis. The FHL Study Group of the Histiocyte Society. Semin Oncol. 1991;18(1):29-33.

11. Henter JI, Arico M, Egeler RM, et al. HLH-94: a treatment protocol for hemophagocytic lymphohistiocytosis. HLH study Group of the Histiocyte Society. Med Pediatr Oncol. 1997;28(5):342-7.

12. Henter JI, Horne A, Arico M, et al. HLH-2004: diagnostic and therapeutic guidelines for hemophagocytic lymphohistiocytosis. Pediatr Blood Cancer. 2007;48(2):124-31.

13. Allen CE, Yu X, Kozinetz CA, McClain KL. Highly elevated ferritin levels and the diagnosis of hemophagocytic lymphohistiocytosis. Pediatr Blood Cancer. 2008;50(6):1227-35.
14. Otrock ZK, Hock KG, Riley SB, de Witte T, Eby CS, Scott MG. Elevated serum ferritin is not specific for hemophagocytic lymphohistiocytosis. Ann Hematol. 2017;96(10):1667-72.

15. Tsuji Y, Ayaki H, Whitman SP, Morrow CS, Torti SV, Torti FM. Coordinate transcriptional and translational regulation of ferritin in response to oxidative stress. Mol Cell Biol. 2000;20(16):5818-27.

16. Diorio C, Henrickson SE, Vella LA, McNerney KO, Chase J, Burudpakdee C, et al. Multisystem inflammatory syndrome in children and COVID-19 are distinct presentations of SARS-CoV-2. J Clin Invest. 2020;130:5967-75.

17. Manson JJ, Crooks C, Naja M, Ledlie A, Goulden B, Liddle T, et al. COVID-19-associated hyperinflammation and escalation of patient care: a retrospective longitudinal cohort study. Lancet Rheumatol. 2020;2(10):e594-602.

18. Schulert GS, Zhang M, Fall N, Husami A, Kissell D, Hanosh A, et al. Whole-exome sequencing reveals mutations in genes linked to hemophagocytic lymphohistiocytosis and macrophage activation syndrome in fatal cases of H1N1 influenza. J Infect Dis. 2016;213(7):1180-8.

19. Zhang K, Biroschak J, Glass DN, Thompson SD, Finkel T, Passo $\mathrm{MH}$, et al. Macrophage activation syndrome in patients with systemic juvenile idiopathic arthritis is associated with MUNC13-4 polymorphisms. Arthritis Rheum. 2008;58(9):2892-6.

20. Lounder DT, Bin Q, de Min C, Jordan MB. Treatment of refractory hemophagocytic lymphohistiocytosis with emapalumab despite severe concurrent infections. Blood Adv. 2019;3(1):47-50.

21. Miettunen PM, Narendran A, Jayanthan A, Behrens EM, Cron RQ. Successful treatment of severe paediatric rheumatic diseaseassociated macrophage activation syndrome with interleukin-1 inhibition following conventional immunosuppressive therapy: case series with 12 patients. Rheumatology (Oxford). 2011;50(2):417-9.

22. Rajasekaran S, Kruse K, Kovey K, Davis AT, Hassan NE, Ndika $\mathrm{AN}$, et al. Therapeutic role of anakinra, an interleukin-1 receptor antagonist, in the management of secondary hemophagocytic lymphohistiocytosis/sepsis/multiple organ dysfunction/ macrophage activating syndrome in critically ill children*. Pediatr Crit Care Med. 2014;15(5):401-8.

23. Goldsmith SR, Saif Ur Rehman S, Shirai CL, Vij K, JF DP. Resolution of secondary hemophagocytic lymphohistiocytosis after treatment with the JAK1/2 inhibitor ruxolitinib. Blood Adv. 2019;3(23):4131-5.

24. Broglie L, Pommert L, Rao S, Thakar M, Phelan R, Margolis D, et al. Ruxolitinib for treatment of refractory hemophagocytic lymphohistiocytosis. Blood Adv. 2017;1(19):1533-6.

25. Albeituni S, Verbist KC, Tedrick PE, Tillman H, Picarsic J, Bassett $\mathrm{R}$, et al. Mechanisms of action of ruxolitinib in murine models of hemophagocytic lymphohistiocytosis. Blood. 2019;134(2):147-59.

Publisher's Note Springer Nature remains neutral with regard to jurisdictional claims in published maps and institutional affiliations. 